\title{
Application of the Theory of a Single First Order Equation to Traffic Flow
}

\author{
Harideo Chaudhary \\ Department of Engineering Science and Humanities, Institute of Engineering, Central Campus, Pulchowk, \\ Tribhuvan University, Nepal \\ Corresponding email: harichau@ hotmail.com
}

\begin{abstract}
Few years ago, Lighthill and Whitham (1955) published a lengthy paper dealing with the theory of highway traffic flow. The basic idea in their approach to the problem is that the flow of traffic along a highway is analogous to the flow of a fluid in an open channel or pipe. This point of view replaces a long column of closely spaced discrete moving vehicles with an equivalent continuous moving stream of liquid (e.g. water) or gas (e.g. air). In other words, Lighthill and Whitham analyzed the phenomenon of traffic flow as though it were a problem in fluid mechanics. This approach allows some, though certainly not all, of the physical and mathematical relationships of hydrodynamics and aerodynamics to be utilized in the traffic flow problem. (Bank, p. 272)
\end{abstract}

Keywords: traffic flow problem; equation of continuity; relation between density-flow; concentration of vehicles; kinematics and characteristic waves.

\section{Introduction}

Traffic flow, in mathematics and civil engineering is the study of interactions between vehicles, drivers and infrastructure (including highways, signage and traffic control devices), with the aim of understanding and developing an optimal road network with efficient movement of traffic and minimal traffic congestion problems. Traffic phenomena are complex and nonlinear depending on the interactions of a large number of vehicles. Due to the individual reactions of human drivers, vehicles do not interact simply following the laws of mechanics, but rather show phenomena of cluster formation and shock wave propagation, both forward and backward, depending on vehicle density in a given area. However, calculations within congested networks are more complex and rely more on empirical studies and extrapolations from actual road counts. Because there are often urban and suburban in nature, other factors (such as road user safety and environmental considerations) also dictate the optimum conditions (wikipedia, p1).

\section{Traffic Stream Properties}

Traffic flow is generally constrained along a one-dimensional pathway (e.g. travel lane). A time space diagram provides a graphical depiction of the flow of vehicles along the pathway over time. Time is measured along the horizontal axis and distance is measured along the vertical axis. Individual trajectory lines of individual vehicles represent traffic flow in a time space diagram. Vehicles following each other along a given travel lane will have a parallel trajectories and trajectories will cross when one vehicle passes another. Time- space diagram is useful tools for displaying and analyzing the traffic flow characteristics of a given roadway segment overtime (e.g. analyzing traffic flow congestion). There are three main variables to visualize a traffic stream: speed $(\mathrm{V})$, density $(\mathrm{K})$ and flow $(\mathrm{Q})$. 


\subsection{Speed}

Speed in traffic flow is defined as the distance covered per unit time. The speed of every vehicle is almost impossible to track on a roadway; therefore, in practice, average speed is based on the sampling of vehicles over a period of time or area and is calculated and used in formulae. If speed is measured by keeping time as reference it is called time mean speed; if it is measured by space reference it is called space mean speed.

Time mean speed is measured by taking a reference area on the roadway over a fixed period of time. In practice it is measured by the use of loop detectors. Vehicles and can track speed of each vehicle. However, average speed measurements obtained from this method are no accurate because instantaneous speed averaged among several vehicles does no account for the difference in travel time for the vehicles that are traveling at different speeds over the same distance

$\mathrm{V}_{\mathrm{t}}=(1 / m) \sum_{i=1}^{m} v_{i}$ where $\mathrm{m}$ represents the number of vehicles passing the fixed point.

Space mean speed is the speed measured by taking the whole roadway segment into account. Consecutive pictures or video of a roadway segment track the speed of individual vehicles, and then the average speed is calculated. It is considered more accurate than the time mean speed. The data for space calculating space mean speed may be taken from satellite pictures, a, camera, or both.

$\mathrm{Vs}=\left(n / \sum_{i=1}^{n} 1 / v_{i}\right)$ where $\mathrm{n}$ represents the number of vehicles passing the roadway segment.

The time mean speed is always greater than space mean speed.

\subsection{Density}

Density $(\mathrm{K})$ is defined as the number of vehicles per unit area of the roadway. In traffic flow, the two most important densities are the critical density $(\mathrm{Kc})$ and jam density $(\mathrm{Kj})$. The maximum density achievable under free flow is $\mathrm{Kc}$, while $\mathrm{Kj}$ is the maximum density achieved under congestion. In general, jam density is seven times the critical density. Inverse of density is spacing(s), which is the center -to -center distance between two vehicles.

$$
\mathrm{K}=1 / \mathrm{s}
$$

The density (k) within a length of roadway (l) at a given time (t) is equal to the inverse of the average spacing of the $\mathrm{n}$ vehicles.

$$
\mathrm{K}(\mathrm{L}, \mathrm{t} 1)=\mathrm{n} / \mathrm{L}=1 / \mathrm{s}(\mathrm{t} 1)
$$

\subsection{Flow}

Flow (Q) is the number of vehicles passing a reference point per unit of time, vehicles per hour. The inverse of flow is headway (h), which is the time that elapses between the ith vehicle passing a reference point in space and the (i+1) th vehicle. In congestion, h remains constant. As traffic jam forms, h approaches infinity.

$$
\mathrm{Q}=\mathrm{KV}
$$




$$
\mathrm{Q}=1 / \mathrm{h}
$$

The flow $(\mathrm{Q})$ passing a fixed point $(\mathrm{x} 1)$ during the interval $(\mathrm{T})$ is equal to the inverse of the average headway of the $\mathrm{m}$ vehicles.

$$
\mathrm{Q}(\mathrm{T}, \mathrm{x} 1)=\mathrm{m} / \mathrm{T} 1=1 / \mathrm{h}(\mathrm{x} 1)
$$

\subsection{Stationary Traffic}

Traffic on a stretch of a road said to be stationary if an observer does not detect movement in an arbitrary area of the time space diagram. Traffic is stationary if all the vehicle trajectories are parallel and equidistant. It is also stationary if it is a superposition of families of trajectories with these properties (e.g. fast and slow drivers).

Obviously, we must include such a microscopic level of observation from the definition and must be satisfied if traffic appears to be similar through larger windows. In fact, we relax the definition even further by only requiring that the quantities $t(A)$ and $d(A)$ be approximately the same, regardless of where the large window (A) is placed.

\section{Derivation of Equation of Continuity}

We begin with concept of conservation of mass. Let us consider the heavy traffic flow on a short stretch of a single lane high way of length $\mathrm{dx}$ as shown in the figure below without entries or exits. Q vehicles per hour are the rate at which vehicles cross the left hand boundary of the stretch. This quantity $\mathrm{Q}$ is called the flux or flow rate. In general, it varies with position $\mathrm{x}$ and time $\mathrm{t}$.

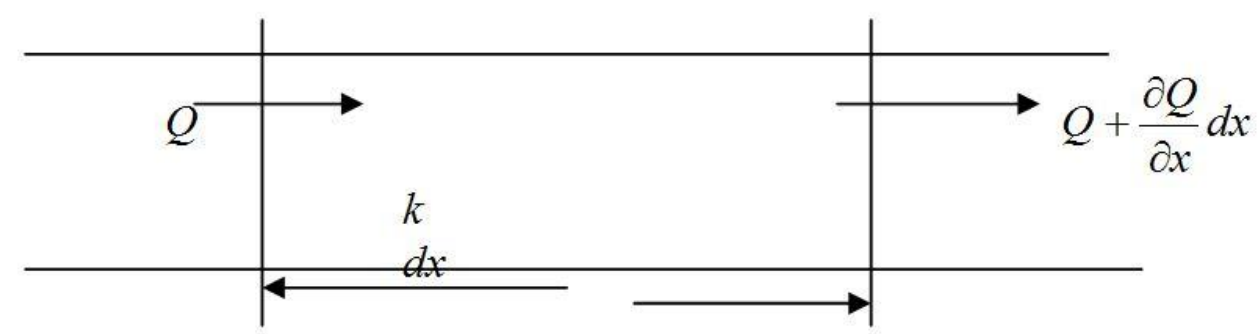

$Q+\frac{\partial Q}{\partial x} d x$ is the rate at which vehicles cross the right hand boundary of the stretch. The total number of cars within two boundaries is given by

$$
Q-\left(Q+\frac{\partial Q}{\partial X} d x\right)
$$

Now the density or concentration of vehicles along the highway is $\mathrm{K}$ vehicles per unit of length (in kilometers). In general this quantity also depends on $\mathrm{x}$ and t. At any instant, the number of vehicles within the stretch of highway $\mathrm{dx}$ is $\mathrm{Kdx}$, and so the rate at which the number of vehicles is accumulating within the stretch is $\frac{\partial(K d x)}{\partial t}$.

From the principle of conservation of mass, we have 


$$
Q-\left(Q+\frac{\partial Q}{\partial X} d x\right)=\frac{\partial K}{\partial t} d x
$$

The terms on the left hand side of this equation are, respectively, the rates at which vehicles are entering and leaving the highway stretch. The right hand side of the equation is the rate at which vehicles are accumulating within the stretch. On simple calculation equation (1) becomes

$$
\frac{\partial Q}{\partial x}+\frac{\partial K}{\partial t}=0
$$

This is partial differential equation in two quantities $\mathrm{Q}$ and $\mathrm{K}$ and called the equation of continuity.

The partial derivatives of this equation indicate there are two independent in the problem, $\mathrm{x}$ and $\mathrm{t}$, and that both of the dependent variables, $\mathrm{Q}$ and $\mathrm{K}$ are functions of $\mathrm{x}$ and t. i.e.

$$
\mathrm{Q}=\mathrm{Q}(\mathrm{x}, \mathrm{t}) \text { and } \mathrm{K}=\mathrm{K}(\mathrm{x}, \mathrm{t})
$$

\section{The Relation between Density and Flow}

In order that traffic flow is fully determined, we need further either a relation between $\mathrm{K}$ and $\mathrm{Q}$ or another differential equation connecting the partial derivatives (Phoolan, p. 50).

To obtain this we put forward the following simplified argument assuming that the highway contains high number of cars it can afford without accidents at any given speed. It is a common experience that in such a traffic movement, smaller the free space between two cars, lower the speed with which they move since there must be sufficient time for the driver to the changes in the speed of the car just ahead. When the space between two cars is zero, i.e. the cars are bumper to bumper, i.e. when $K=K_{\max }$, the flow velocity must be zero. Therefore to the first approximation we can assume the flow velocity, i.e. the velocity $\mathrm{V}$ of the cars to be a function of the local density, i.e. $\mathrm{V}=\mathrm{V}(\mathrm{K})$ (Phoolan, p51) where $\mathrm{V}(\mathrm{K})$ is a decreasing function of $\mathrm{K}$ and $\mathrm{V}$ $\left(\mathrm{K}_{\max }\right)=0$. The flux $\mathrm{Q}$ is also a function of $\mathrm{K}$ and related to $\mathrm{V}$ by

$$
V=\frac{Q}{K}
$$

We can write,

$$
\mathrm{Q}=\mathrm{VK}
$$

This indicates that the flow $\mathrm{Q}$ is equal to the product of the velocity $\mathrm{V}$ and concentration or density K. Further when the road is free, there is maximum velocity with which the cars can move, i.e. as $\mathrm{K}$ tends to zero, $\mathrm{V}$ attain maximum value.

\section{The Fundamental Diagram of Traffic Flow}

Numerous field studies have shown that the velocity $\mathrm{V}$ of a stream of traffic on a highway decreases as the concentration of vehicles, $\mathrm{K}$ increases. We can develop the simple linear relationship between $\mathrm{V}$ and $\mathrm{K}$ as 


$$
V=V_{\max }\left(1-\frac{K}{K_{\max }}\right)
$$

Here $\mathrm{V}_{\max }$ is the maximum velocity (perhaps set by the speed limit or weather conditions) and $\mathrm{K}_{\max }$ is the jam concentration (Bank, p274). We can say from the above relation that when the concentration $K=0$, the velocity $V$ is a maximum, and when the concentration $K=K_{\max }$ (bumper to bumper), the velocity is zero.

From equations (4) and (5), we get

$$
Q=V_{\max } K\left(1-\frac{K}{K_{\max }}\right)
$$

This is called Equation of state. (Bank, p276)

From the relation (6) it is clear that flow $\mathrm{Q}$ becomes zero when $\mathrm{K}=0$ i.e. there are no vehicles on the highway. When $\mathrm{K}=\mathrm{K}_{\max }$ the traffic is completely jammed and stalled and the velocity becomes zero in this case

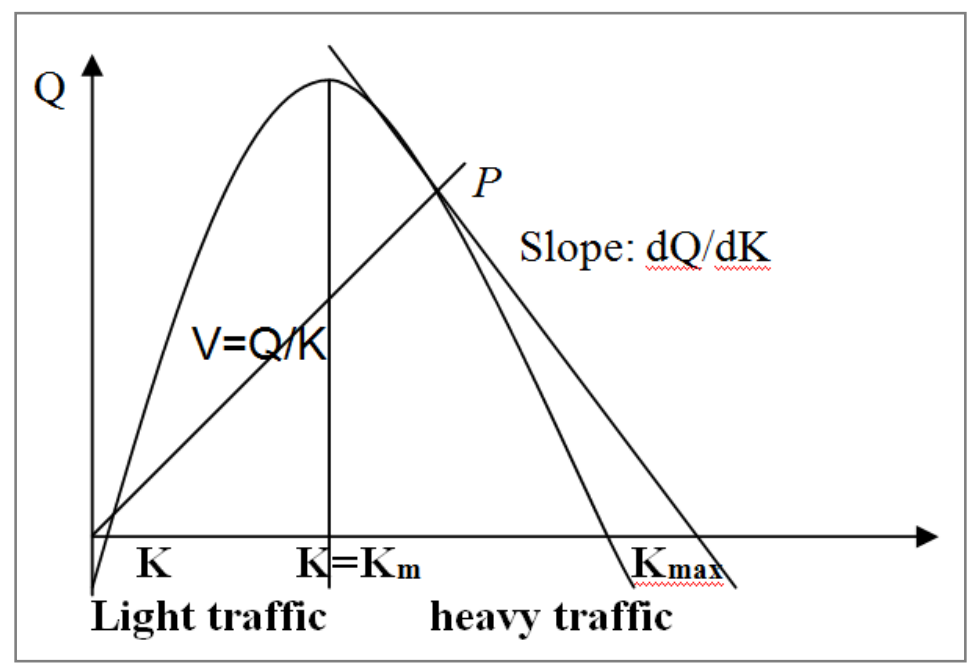

Flow-Density curve

Figure shows the relationship between $\mathrm{Q}$ and $\mathrm{K}$. This relationship is known as Fundamental diagram of traffic flow (Bank, p275). The function $\mathrm{Q}$ has maximum value for some value of $\mathrm{K}$, say $\mathrm{K}=\mathrm{K}_{\mathrm{m}}$.

\section{Kinematical Waves and Characteristic Curves}

We can rewrite the equation (2) as

$$
\frac{\partial K}{\partial t}+\frac{d Q}{d K} \frac{\partial K}{\partial x}=0
$$

We can write, 


$$
\frac{\partial K}{\partial t}+C \frac{\partial K}{\partial x}=0
$$

Where $C=\frac{d Q}{d K}$ is the velocity of kinetic wave moving through the stream of vehicles.

Equation (8) is known as the nonlinear first order partial differential equation (Bank, p277).

Differentiating (6), we get

$$
C=\frac{d Q}{d K}=V_{\max }\left(1-\frac{2 K}{K_{\max }}\right)
$$

Also we have

$$
C=\frac{d Q}{d K}=d(V K)=V+K \frac{d V}{d K}
$$

Any acceleration or deceleration of a car is felt by other drivers and its effect travels with the "sound speed" C given by (10). Since $\mathrm{V}$ is a decreasing function of $\mathrm{K}, \frac{d V}{d K}<0$ and hence the velocity of propagation relative to the velocity of the traffic is negative, i.e. the effect is felt by the drivers of the following cars.

\section{Conclusion}

Let $\mathrm{P}$ be any point on the flow-density curve. The slope of the radius vector from the origin to point $\mathrm{P}$ gives the velocity of the vehicles, $\mathrm{V}=\mathrm{Q} / \mathrm{K}$. Similarly the slope of the tangent to the curve at point $\mathrm{P}$ gives the wave velocity, $C=\frac{d Q}{d K}$. From the figure, we note that

(i) If $\mathrm{K}<\mathrm{K}_{\mathrm{m}}$ (i.e. light traffic), the slope is positive and so the wave velocity is positive. Accordingly, the wave moves downstream with respect to the highway (i.e. in the direction of traffic flow).

(ii) If $K>K_{m}$ (i.e. heavy traffic), the slope and wave velocity are negative. Hence the wave moves upstream with respect to the highway (i.e. in the direction opposite to traffic flow).

(iii) If $\mathrm{K}=\mathrm{K}_{\mathrm{m}}$ the wave is stationary. In this regime the flow rate is maximal.

The equation (8) is known as a nonlinear first order partial differential equation. The general solution to this equation is

$$
\mathrm{K}=\mathrm{f}(\mathrm{x}-\mathrm{Ct})
$$

Thus the concentration $\mathrm{K}$ is a function of the quantity $(\mathrm{x}-\mathrm{Ct})$ The quantity represents a so called kinematical wave moving with velocity $\mathrm{C}$ corresponding to a particular value of $\mathrm{C}$ 


\section{References}

[1] Phoolan Prasad, Renuka Ravindran, Partial Differential Equations, New Age International Publishers, First edition, 1985

[2] Robert B. Banks, Towing icebergs, Falling Dominoes, and other Adventures in Applied Mathematics, University press (India) limited 3-5-819 hyderguda, Hyderabad 500029

[3] Traffic Flow from wikipedia, the free encylopedia

[4] Madan M. Pradhan, Traffic Engineering, vol. 1, Joshi Printing Press, kwako, Lalitpur, Nepal, 2005

[5] Lokenath Debnath, Non-linear partial differential equations for scientists and engineers, Third edition, Springer, October 7, 2011. 